\title{
A ROCKET PAYLOAD USING CASSEgRAin-ECHELle OPTICS WITH IMAGE INTENSIFICATION FOR HIGH RESOLUTION ULTRAVIOLET STELLAR SPECTROSCOPY
}

\author{
W. M. BURTON, N. K. REAY, D. B. SHENTON, and R. WILSON \\ Astrophysics Research Unit, Culham Laboratory, Abingdon, Berkshire, England
}

\begin{abstract}
A description is given of a rocket payload designed to record high resolution ultraviolet stellar spectra using the star-stabilised 'Skylark' rocket vehicle. The system consists of a $35 \mathrm{~cm}$ aperture $f / 12$ Cassegrain telescope and a spectrometer in which the high spectral dispersion of an echelle grating is crossed with that of a plane grating to separate the echelle orders. The spectrum is focussed onto an electrostatic image intensifier tube, the fibre optic faceplate of which is coated with sodium salicylate to give ultraviolet sensitivity. Photographic film is used to record the two dimensional spectrum array with a resolving power $\lambda / \mathrm{d} \lambda \simeq 10^{4}$ between $1200 \AA$ and $3000 \AA$. Efficiency measurements of components indicate that the photometric efficiency of the entire system will allow spectra to be recorded for early-type stars which are brighter than $m_{r} \simeq 5$.
\end{abstract}

\section{Introduction}

Many important discoveries have been made in recent years as a result of ultraviolet astronomical observations from rocket and satellite vehicles. Measurements of the density of interstellar atomic and molecular hydrogen, the extinction of interstellar dust, the mass loss-rate in early-type supergiants and the observation of ultraviolet emission from nebulae are all of great astrophysical significance. To extend these observations, one clear need is to record stellar ultraviolet spectra with increased spectral resolution so that the rich spectra can be studied in detail. For this reason, a rocket payload has been designed to provide suitable instrumentation for high resolution stellar spectroscopic studies. The specification adopted for this payload required a spectral resolving power $\lambda / \mathrm{d} \lambda \simeq 10^{4}$ over the wavelength range $1200-3000 \AA$ for stars brighter than $m_{v} \simeq 5$.

\section{Payload Design}

The Culham astronomy programme is based on the star-stabilised 'Skylark' rocket, a $44 \mathrm{~cm}$ diameter 2-stage solid fuel vehicle which can achieve an altitude of more than $200 \mathrm{~km}$. The stellar stabilisation system developed for the U.K. Science Research Council sounding rocket programme by GEC-Elliott Ltd., Frimley is designed to point the rocket payload towards a selected star of visual magnitude $m_{v} \approx 5$ with an accuracy of a few arc sec for a typical flight duration of four minutes (Walker and Abbott, 1970). A basic payload has been designed as part of the star-pointed 'Skylark' system. the intention being that similar payloads will be flown regularly with little modification and thus form the basis of a continuing UV astronomy programme.

A Cassegrain telescope is used to focus a stellar image onto the entrance aperture of an echelle spectrograph which contains an image intensifier and photographic film 
for data recording. Figures 1 and 2 show the payload design concept. The dispersion of the echelle grating is crossed with that of a plane grating to separate the orders in the echelle spectrum. An echelle system was chosen for this design

(a) because its high angular dispersion allowed all dimensional tolerances (mechanical, thermal and stabilisation) to be relieved and

(b) because the two-dimensional spectrum format is ideally suited to image tube detection which gives a consequent gain in sensitivity.

\subsection{TELESCOPE}

The telescope is of classical Cassegrain design $(f / 12)$ with a $35 \mathrm{~cm}$ aperture $f / 2.6$ parabolic primary and a $7.2 \mathrm{~cm}$ diameter hyperbolic secondary mirror. The secondary mirror support structure is made from titanium which is both light and strong and the optical components from low expansion glass (Schott Duran 50). Thermal effects within the telescope must be considered as the rocket skin temperature may increase by $150 \mathrm{C}$ during flight. However, the thermal inertia of the telescope system and the short flight duration will allow only a limited temperature rise on the telescope tube and primary mirror. This can be further reduced by thermally insulating the rocket skin from the telescope structure and the primary mirror clamping points.

In flight, it is not possible to 'compensate' the telescope by matching the expansion coefficients of the structure and the optical components, since compensation is only achieved when the temperature changes uniformly throughout the entire telescope system. In practice this is unlikely because of the short duration of the flight and the localised heat inputs at the mirror clamping points.

\subsection{STAR ALIGNMENT SENSOR}

The star alignment sensor is located near to the telescope focus and is fixed rigidly to the telescope structure, forming a unit to which any suitable designed spectrometer can be attached.

A $9.2 \mathrm{~cm}$ diameter conical mirror, placed at $45^{\circ}$ to the incident light just forward of the telescope focal plane, diverts starlight into the alignment sensor. A small hole at the vertex of the conical mirror allows light to pass through to the spectrograph entrance aperture which is attached to the rear surface of the mirror. When the vehicle is stabilised and pointed towards a star, the defocussed light patch falls symmetrically on the hole in the center of the conical mirror and a narrow annulus is diverted into the error sensor. A rotating aperture modulates the signal produced by a photomultiplier tube illuminated by the starlight and an error signal is generated when the light distribution ceases to be symmetric about the hole in the conical mirror vertex. The field of view of the star sensor is $10 \times 0.5$ degrees and the typical pointing noise is expected to be approximately $2.5 \mathrm{arcsec}(\mathrm{rms})$.

When the rocket is launched the method of acquisition on the star is as follows. After separation of the rocket motor, a moon sensor mounted forward of the telescope secondary mirror allows the payload to be pointed towards the moon. The payload then executes a roll manoeuvre by sensing the earth's magnetic field to set the 


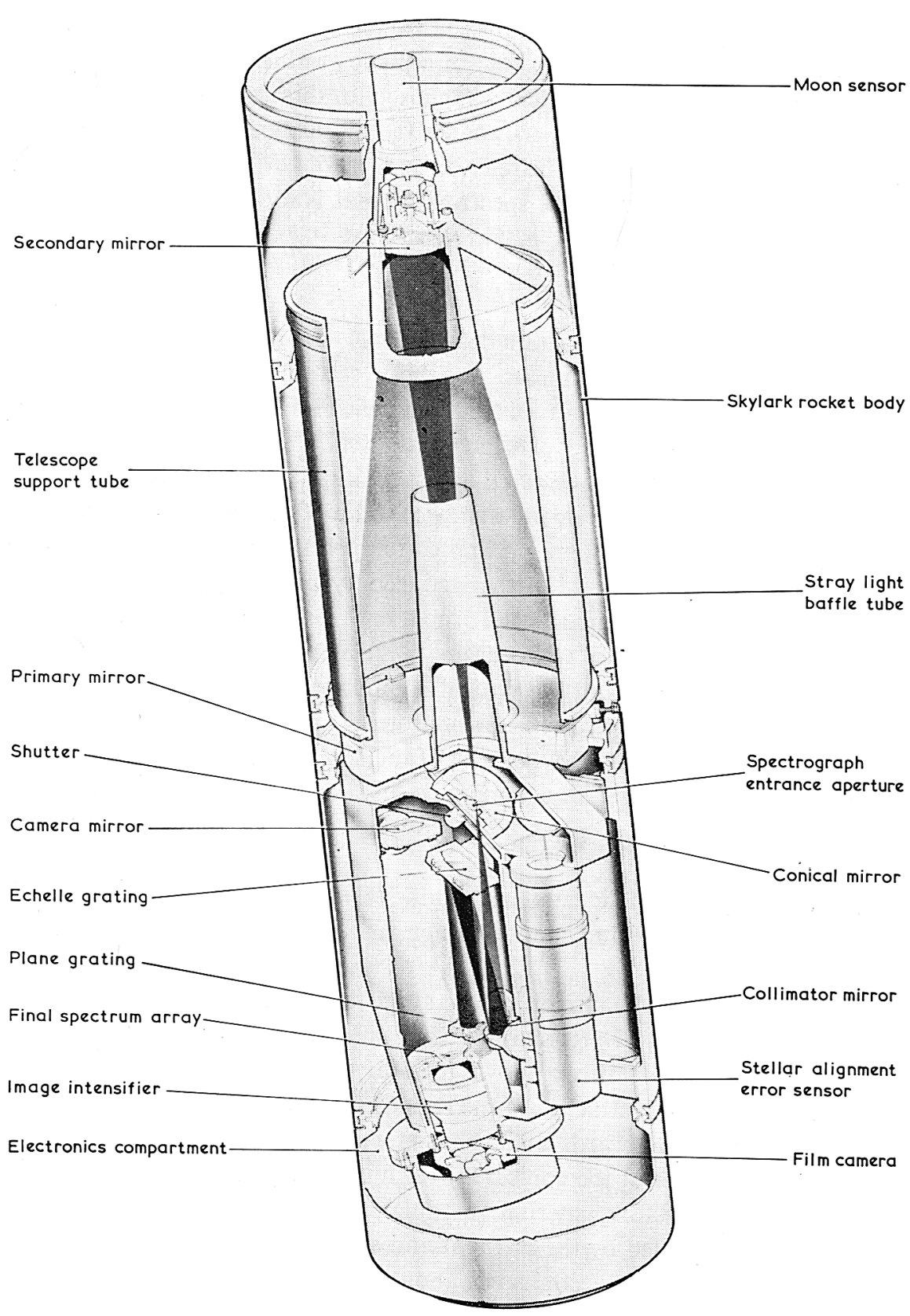

Fig. 1. Schematic view of Cassegrain-echelle optical system. 
A ROCKLT PAYLOAD USING ('ASSEGRAIN-ECHELLE OPTIC'S

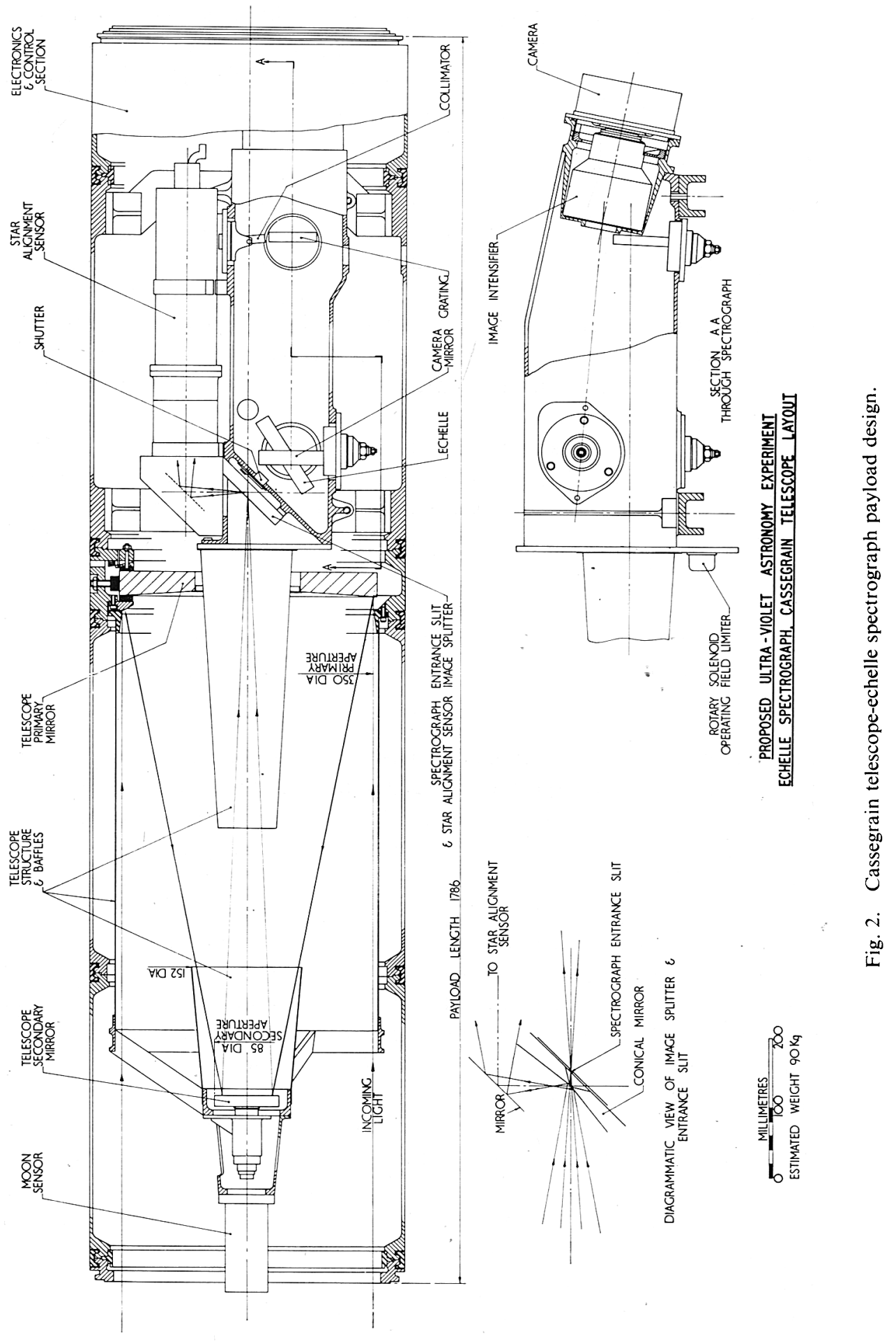


selected star, the moon and the payload slew jets into the same plane. A pre-programmed slew manoeuvre controlled by an integrating rate-gyro then brings the star into the field of view of the telescope, at which time control is passed to the star alignment sensor to complete the acquisition process.

\subsection{SPECTROGRAPH}

The spectrograph uses four separate reflecting optical components. Light entering the spectrograph slit is collimated by an off-axis paraboloid mirror and then illuminates an echelle grating which has 73 line $\mathrm{mm}^{-1}$ and a blaze angle of 63 . A plane grating with 600 line $\mathrm{mm}^{-1}$ is placed with its plane of dispersion perpendicular to that of the echelle, so that the crossed dispersion separates the orders in the echelle spectrum. The dispersed collimated light is focussed by a spherical concave mirror to form a two dimensional spectrum array on the input faceplate of an image intensifier tube. Both the echelle and the plane grating are replica rulings supplied by Bausch and Lomb. The inherent high angular dispersion of the echelle allows a relaxation in the accuracy requirement of the star pointing system for a given spectral resolution, the entrance slit dimensions $(80 \times 50 \mu \mathrm{m})$ having equivalent star field angles of $4 \times 2.5$ arcsec.

The spectral format is as shown in Figure 3. The spectral resolution varies with wavelength as follows:

$\begin{array}{llll}\text { wavelength }(\AA) & 1200 & 2100 & 3000 \\ \text { spectral resolution } & 0.07 \AA & 0.15 \AA & 0.27 \AA\end{array}$

A spherical camera mirror was chosen because it introduces astigmatism into the spectrum, which elongates the spectral lines and so facilitates subsequent photometry. If the position of the focal plane is chosen carefully, this can be done with very little degradation of the spectral resolution. The results of ray trace calculations to estimate image quality are shown in Figure 4 for the optimum focal plane position, while Figure 5 shows the cycle separation and the calculated spectral line lengths for both spherical and paraboloidal camera mirrors. Defining the 'useful' aberrational line length as that corresponding to the specified spectral resolution line width, then curve ' $e$ ' in Figure 5 shows that the useful line length increases smoothly with wavelength. The total aberrational line length (including the flare) is less than the cycle spacing, so that overlapping does not occur and the flare provides a graded 'exposure wedge', albeit at reduced spectral resolution.

An important factor in determining the efficiency of the complete system is the reflectance of the optics. All of the optical components in the telescope and spectrograph are aluminised and overcoated with magnesium fluoride. Mirrors are coated in the laboratory and have a measured reflectance of at least $65 \%$ at $1216 \AA\left(\mathrm{H}_{\mathrm{L}} \alpha\right)$. Gratings are coated by the manufacturer and efficiency measurements are made in the laboratory. Figure 6 shows efficiency curves for four echelle gratings from two different master rulings and also a typical mirror reflectivity curve (Burton and Reay. 


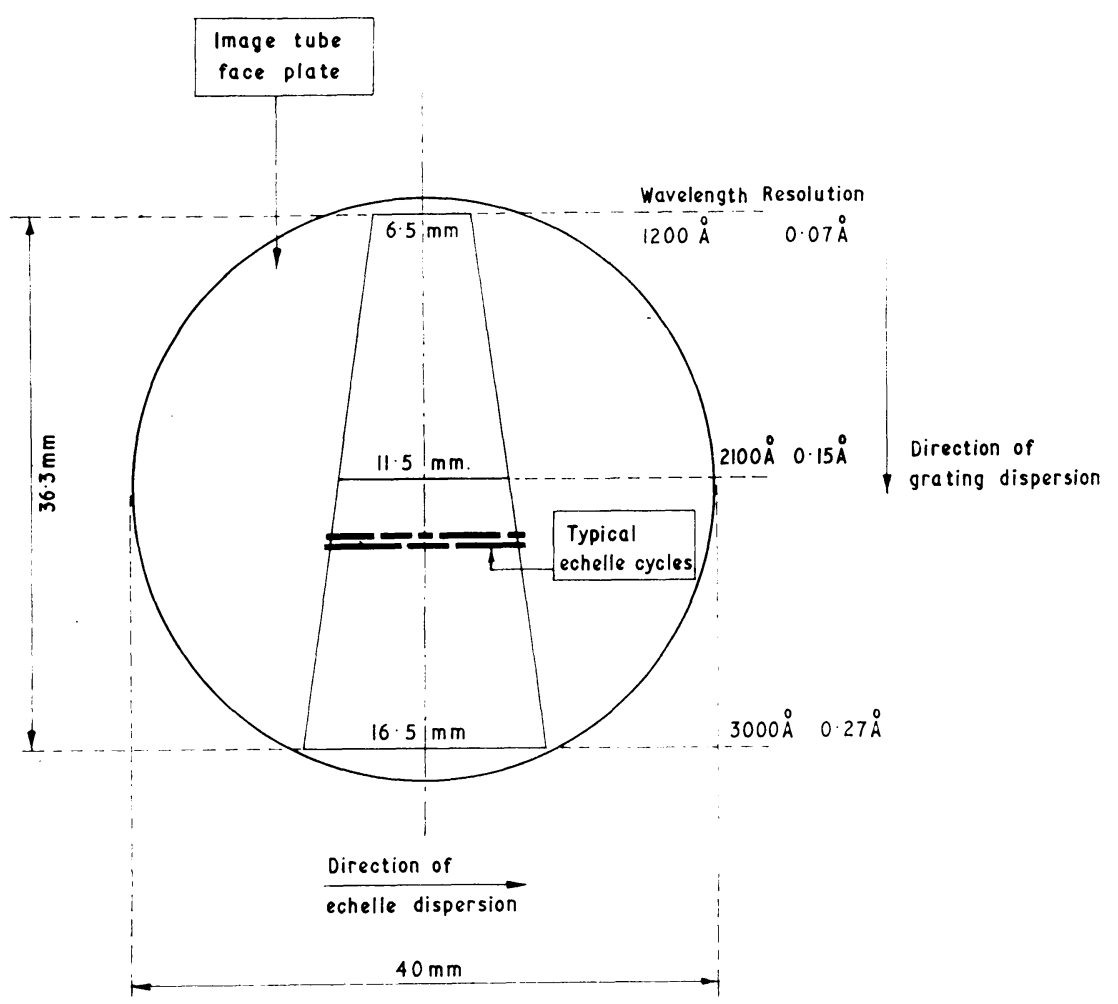

Fig. 3. Spectrum format on the input window of the image intensifier tube.

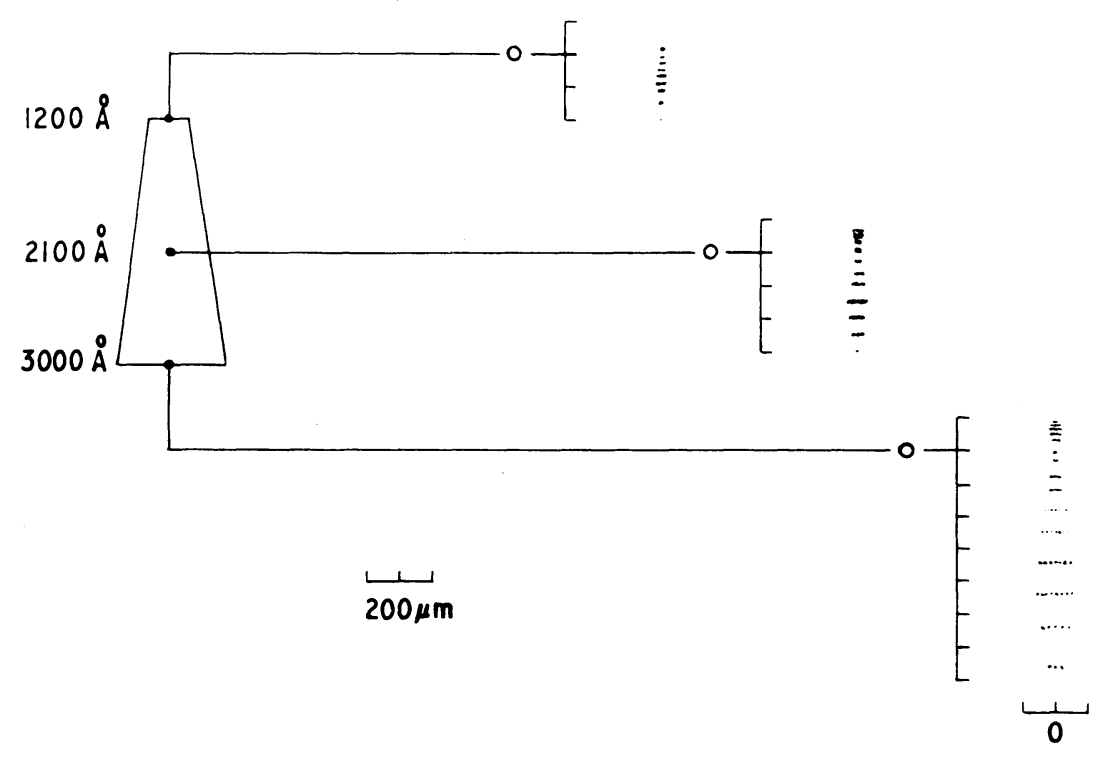

Fig. 4. Computed spectral line images in the focal plane of a spherical concave camera mirror. 


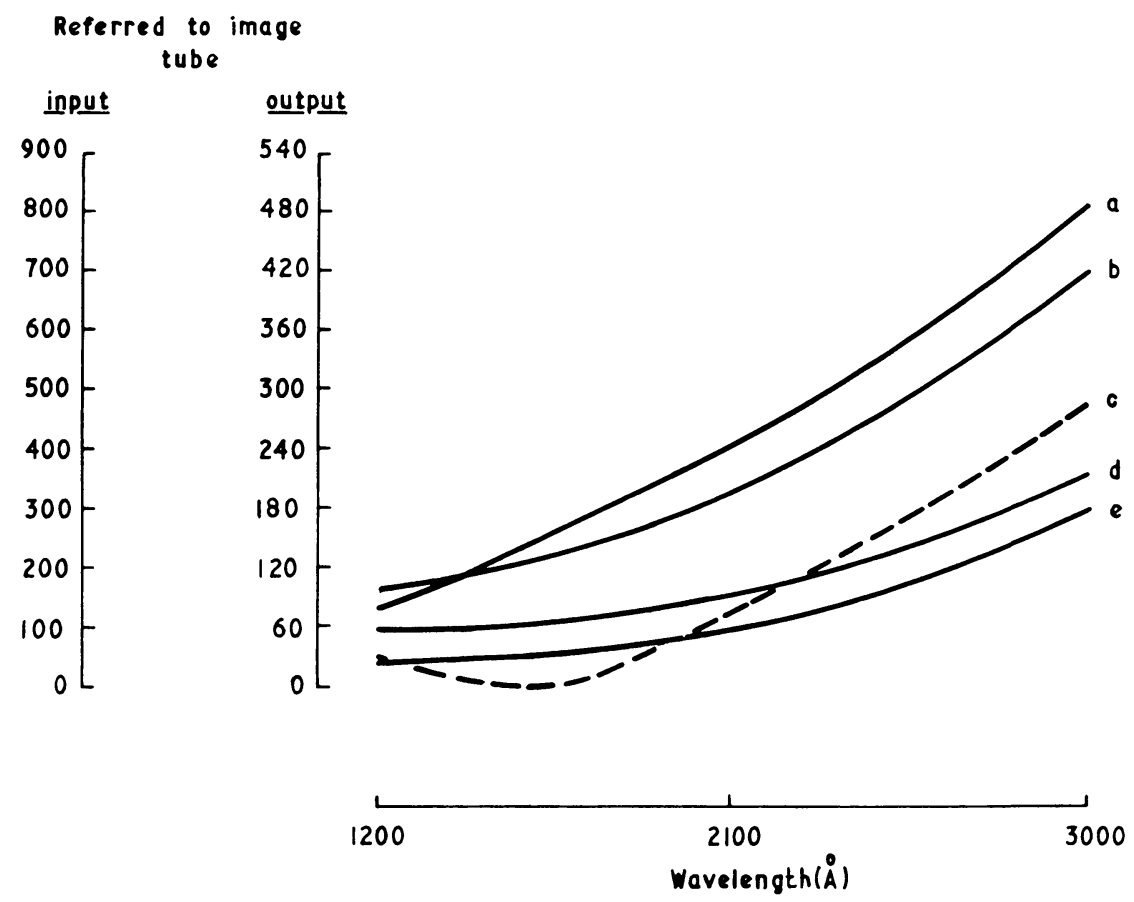

Fig. 5. Echelle cycle separation and spectral line length as a function of wavelength. (a) echelle cycle separation; (b) aberrational line length from spherical camera mirror; (c) aberrational line length from paraboloid camera mirror; (d) total spectral line length from spherical mirror (geometric image ' 'useful' aberrational length); and (e) 'useful' aberrational line length.

(All values are given in $\mu \mathrm{m}$ units.)

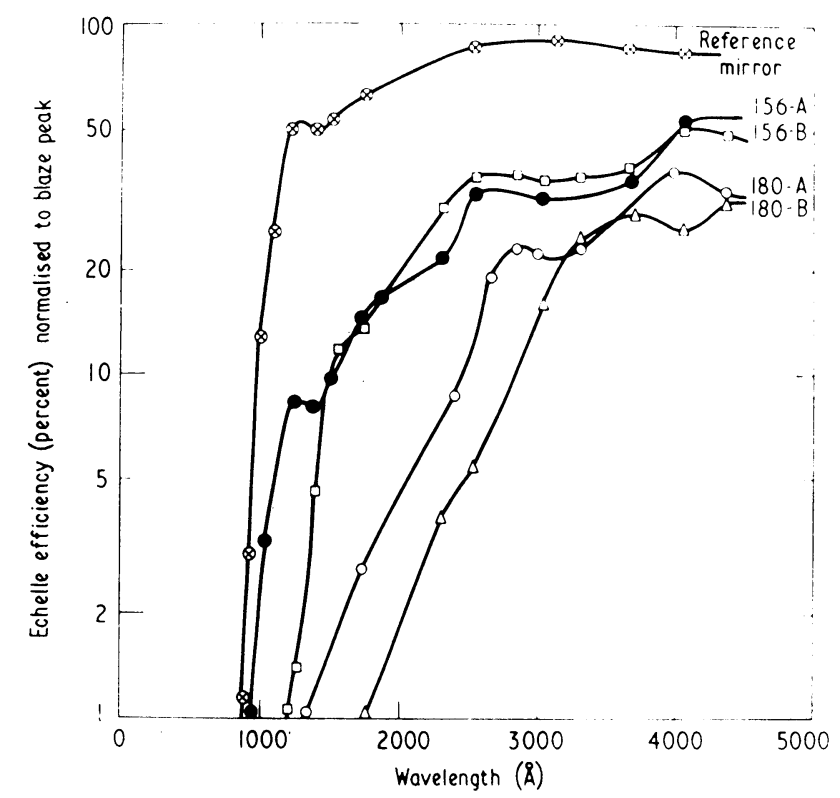

Fig. 6. Echelle efficiency measurements normalized to peak blaze as a function of wavelength. The upper curve shows the measured reflectance of a reference mirror coated with $\mathrm{Al}-\mathrm{MgF}_{2}$. 
1970). Clearly, echelle ruling No. 156 is superior in terms of efficiency to ruling No. 180. As the echelle is not being used at anything approaching limiting spectral resolution, the quality of the echelle grating in this respect is less important.

\subsection{Detector SySTEM}

In order to increase the sensitivity of the instrument, a fibre optic coupled image intensifier tube (Westinghouse Type WL30677) increases the intensity of the spectrum before it is recorded on film. The tube is sensitised for ultraviolet detection by coating the input fibre optic window with a thin layer of sodium salicylate, the emerging visible light being recorded on Kodak 103a-F film. The $40 \mathrm{~mm}$ diameter input faceplate is just large enough to accommodate the spectrum array (see Figure 3 ) and the demagnification factor $\times 0.64$ produces an output image suitable for recording on $35 \mathrm{~mm}$ film.

Laboratory measurements to evaluate the image tube performance in terms of its

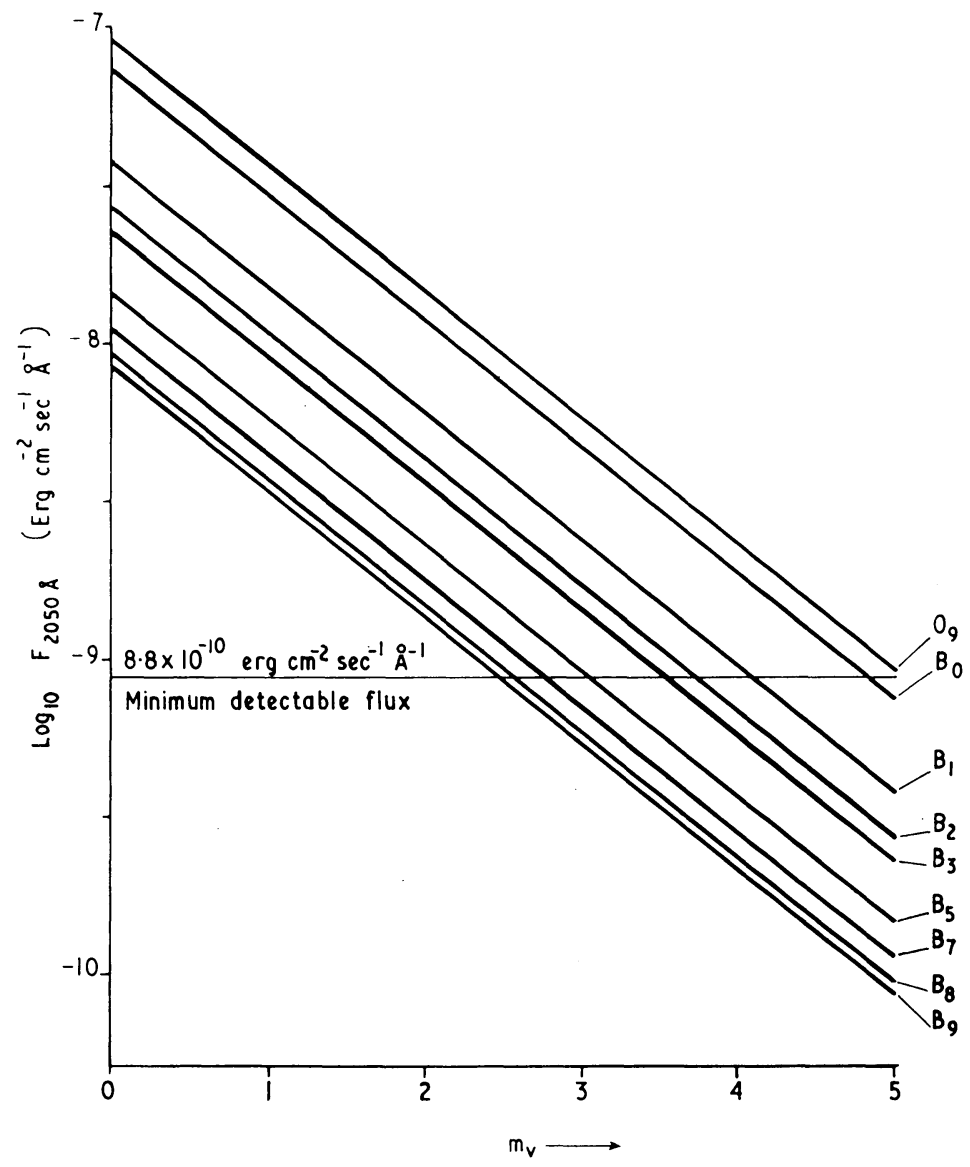

Fig. 7. Estimated limiting stellar magnitudes for observation of spectra at $2050 \AA$ calculated for stars of different spectral type. 
suitability for use as a UV detector are now in progress, but at the present time only results of a preliminary nature are available. They indicate that the tube will increase the sensitivity of the instrument by a factor of ten $\left(\Delta m_{v} \simeq 2.5\right)$ relative to an alternative detector system using the fastest available ultraviolet films (Kodak-Pathé SC5 and SC7).

The measurements of detector sensitivity and optical component efficiency allow calculations to be made of the limiting stellar magnitude for which spectra can be observed (Macchetto, 1970). Figure 7 shows the results of these calculations for stars of different spectral type. The limiting magnitudes are based on predicted values for the ultraviolet emission and they assume a minimum detectable density (0.1D) image obtained during an exposure time of $100 \mathrm{~s}$.

Payload design work is well advanced at this time and many of the components are being manufactured. Payload integration will begin in 1971 and the first flight is scheduled to take place early in 1972. Three flights are provided for initially, with a further one or two each year during the period 1973-75.

\section{Acknowledgements}

Important contributions to the work described in this paper have been made by Dr A. Boksenberg (University College, London), Mr I. Walker and Mr D. Milton (GEC - Elliott Ltd., Frimley) and by our colleagues at Culham Laboratory, $\mathrm{Mr}$ W. G. Griffin, Mr C. Lewis, Dr F. Macchetto, Mr H. J. B. Paxton and Mr B. A. Powell. The initial payload design was carried out by Mr P. J. Barker and his colleagues at AWRE, Aldermaston.

\section{References}

Burton, W. M. and Reay, N. K.: 1970, Appl. Opt.9, 1227.

Macchetto, F. M. F.: 1970, (private communication).

Walker, I. F. M. and Abbot, J. K.: 1970 'A Star-Pointing attitude Control System for the Skylark Sounding Rocket'. Paper to be presented at the 'Sounding Rocket Vehicle Technology Specialist Conference', Williamsburg, Virginia, U.S.A., December 1970.

\section{DISCUSSION}

R. Muri: What is the absolute spatial resolution?

W. M. Burton: The Westinghouse WL 30677 image intensifier has a resolution of 10 line $\mathrm{pair} / \mathrm{mm}$ for $70 \%$ modulation of a square wave pattern. This is not significantly degraded by the sodium salicylate coating.

R. Muri: Did you use magnetical or electrostatic image optics?

$W . M$. Burton: The image intensifier has electrostatic focussing.

$R$.J. Van Duinen: What is the diameter of the fibres?

W. M. Burton: The input faceplate has fibres of $16 \mu \mathrm{m}$ diameter.

G. Courtès: Did you consider the possibility to use crossed dispersion owing to a ruled secondary mirror of the telescope or a ruled collimator? The economy could be of one or perhaps two reflexions.

W.M. Burton: Other configurations were considered. The adopted design has the important advantage that the detector unit is located at the opposite end of the spectrometer from the slit. This removes the difficulty of fitting the detector close to the telescope primary mirror and the star alignment error sensor. We could not introduce dispersion in the telescope because the alignment system prevents this. 